\title{
CRITERIA FOR FUNCTIONS TO BE OF HARDY CLASS $H^{p}$
}

\author{
SHINJI YAMASHITA
}

\begin{abstract}
Let $f$ be holomorphic in the disk $|z|<1$. Two criteria (see (I) and (II)) for $f$ to be of $H^{2}$ are extended to the case of $H^{p}, 0<p<+\infty$, by the methods different from known ones for $p=2$.
\end{abstract}

1. Results. By $f$ we always mean a function holomorphic in $D=\{|z|<1\}$. We set for $0<p<+\infty$,

$$
f_{p}^{*}=\frac{p}{2}|f|^{p / 2-1}\left|f^{\prime}\right|
$$

roughly speaking, $f_{p}^{*}$ is the absolute value of the derivative of $f^{p / 2}$ which has no meaning when $f$ has a zero in $D$. Obviously, $f_{2}^{*}=\left|f^{\prime}\right|$. We shall show that $f_{p}^{*}$, which may assume the value $+\infty$, plays important roles for $f$ to be of class $H^{p}(0<p<+\infty)$ in $D$. Here, $f$ is said to be of Hardy class $H^{p}(0<p<+$ $\infty)$ if

$$
I_{p}(r, f)=\frac{1}{2 \pi} \int_{0}^{2 \pi}\left|f\left(r e^{i \theta}\right)\right|^{p} d \theta
$$

is bounded for $0<r<1$ (see [1, p. 2]). Set

$$
A_{p}(f)=\iint_{D}(1-|z|) f_{p}^{*}(z)^{2} d x d y \quad(z=x+\dot{y}) .
$$

The following (I) is observed in [5, Remark (a), p. 208].

(I) $f$ is of class $H^{2}$ if and only if $A_{2}(f)<+\infty$.

This is the case $p=2$ in our

THEOREM 1. Let $f$ be a function holomorphic in $D$, and let $0<p<+\infty$. Then $f$ is of class $H^{p}$ if and only if $A_{p}(f)<+\infty$.

Our proof of Theorem 1 is different from that of (I) (see [5, p. 208]) where the coefficients of the Taylor expansion of $f$ about 0 play the essential roles.

Now, let $G$ be a subdomain of $D$ such that the boundary of $G$ has the only one point 1 in common with the unit circle. Assume that there exists $r_{0}$, $0<r_{0}<1$, depending on $G$, such that the intersection of $G$ with each circle $\{|z|=r\}, r_{0}<r<1$, is of linear measure $r \varphi(r)$, where

$$
\liminf _{r \rightarrow 1}(1-r)^{-1} \varphi(r)>0
$$

Received by the editors May 23, 1978.

AMS (MOS) subject classifications (1970). Primary 30A78. 
and

$$
\limsup _{r \rightarrow 1}(1-r)^{-1} \varphi(r)<+\infty
$$

Let $\mathcal{G}$ be the family of all domains $G$ of the type described above. A typical example of $G$ is a triangular domain in $D$ with one vertex at 1 , which we shall call, for short, a triangular domain at 1 . Of course, $G \in \mathcal{G}$ may look like a "Swiss cheese", having many holes. Denoting

$$
G(\theta)=\left\{z \in D \mid e^{-i \theta} z \in G\right\}, \quad \theta \in[0,2 \pi],
$$

we say that $f$ satisfies the $p$-Lusin property with respect to $G \in \mathcal{G}$ if

$$
L_{p}(f, G, \theta)=\iint_{G(\theta)} f_{p}^{*}(z)^{2} d x d y
$$

is summable with respect to $\theta$ on $[0,2 \pi](0<p<+\infty)$; thus, it follows that $L_{p}(f, G, \theta)<+\infty$ for almost every $\theta$ in $[0,2 \pi]$.

G. Piranian and W. Rudin [4, Theorem 1] reformulated (in effect, with an addition) N. Lusin's theorem [3] as follows.

(II) If $f \in H^{2}$, then $f$ has the 2-Lusin property with respect to each triangular domain at 1 . Conversely, if $f$ has the 2-Lusin property with respect to a certain triangular domain at 1 , then $f \in H^{2}$.

Again, this is the case $p=2$ in our

THEOREM 2. Let $f$ be a function holomorphic in $D$, and let $0<p<+\infty$. If $f \in H^{p}$, then $f$ has the $p$-Lusin property with respect to each domain of class $\mathcal{G}$. Conversely, if $f$ has the $p$-Lusin property with respect to a certain domain of $\mathcal{G}$, then $f \in H^{p}$.

Our proof of Theorem 2 is different from that of (II) (see [3] and [4]) where the coefficients of the Taylor expansion of $f$ about 0 play the essential roles.

2. Proof of Theorem 1. First of all, the local consideration of $f_{p}^{*}$, near the zeros of $f$, shows that for each $0<p<+\infty$ and for each $0<r<1$,

$$
E_{p}(r, f)=\iint_{|z|<r} f_{p}^{*}(z)^{2} d x d y<+\infty .
$$

In effect, G. H. Hardy and P. Stein (see [2, Theorem 3.1, p. 42]) proved much more:

$$
r \frac{d}{d r} I_{p}(r, f)=\frac{2}{\pi} E_{p}(r, f), \quad 0<r<1 .
$$

It then follows from the integration that

$$
I_{p}(r, f)-|f(0)|^{p}=\frac{2}{\pi} \int_{0}^{r} E_{p}(t, f) t^{-1} d t, \quad 0<r<1 .
$$

Especially,

$$
\int_{0}^{r} E_{p}(t, f) t^{-1} d t<+\infty
$$


for each $0<r<1$. Now, it follows from (2.1) that, $f \in H^{p}$ if and only if

$$
\int_{0}^{1} E_{p}(t, f) t^{-1} d t<+\infty .
$$

In view of (2.2) one can conclude from (2.3) that, $f \in H^{p}$ if and only if

$$
\int_{0}^{1} E_{p}(r, f) d r<+\infty \text {. }
$$

Now, letting $X_{r}(z)$ be the characteristic function of the disk $\{|z|<r\}$, $0<r<1$, one observes that

$$
\int_{0}^{1} X_{r}(z) d r=1-|z|, \quad z \in D
$$

whence

$$
\begin{aligned}
\int_{0}^{1} E_{p}(r, f) d r & =\int_{0}^{1} d r \iint_{D} f_{p}^{*}(z)^{2} X_{r}(z) d x d y \\
& =\iint_{D}\left[\int_{0}^{1} X_{r}(z) d r\right] f_{p}^{*}(z)^{2} d x d y=A_{p}(f) .
\end{aligned}
$$

This equality, together with (2.4), completes the proof of Theorem 1 .

3. Proof of Theorem 2. Let $G \in \mathcal{G}$ with $r_{0}$ and $\varphi$ as described in the definition of $G$. Letting $X(z, \theta)$ be the characteristic function of $G(\theta)$, $\theta \in[0,2 \pi]$, one observes that

$$
\int_{0}^{2 \pi} X(z, \theta) d \theta, \quad z \in D,
$$

is the linear measure of the set $\left\{e^{i \theta} \mid z \in G(\theta)\right\}$. Therefore,

$$
\varphi(|z|)=\int_{0}^{2 \pi} X(z, \theta) d \theta, \quad r_{0}<|z|<1 .
$$

On the other hand,

$$
\begin{aligned}
L_{p}(f, G) & \equiv \int_{0}^{2 \pi} L_{p}(f, G, \theta) d \theta \\
& =\iint_{D}\left[\int_{0}^{2 \pi} X(z, \theta) d \theta\right] f_{p}^{*}(z)^{2} d x d y .
\end{aligned}
$$

It follows from (1.1), (1.2), (3.1), and (3.2) that $L_{p}(f, G)<+\infty$ if and only if there exists $r_{2}, r_{0}<r_{2}<1$, such that

$$
\iint_{r_{2}<|z|<1}(1-|z|) f_{p}^{*}(z)^{2} d x d y<+\infty
$$

Since (3.3) is equivalent to $A_{p}(f)<+\infty$, Theorem 2 now follows from Theorem 1. 


\section{REFERENCES}

1. P. L. Duren, Theory of $H^{p}$ spaces, Academic Press, New York and London, 1970.

2. W. K. Hayman, Multivalent functions, Cambridge Univ. Press, London and New York, 1958.

3. N. Lusin, Sur une propriété des fonctions à carré sommable, Bull. Calcutta Math. Soc. 20 (1930), 139-154.

4. G. Piranian and W. Rudin, Lusin's theorem on areas of conformal maps, Michigan Math. J. 3 (1955-1956), 191-199.

5. A. Zygmund, Trigonometric series. II, Cambridge Univ. Press, London and New York, 1959.

Department of Mathematics, Tokyo Metropoutan University, Furazawa, Setagaya-kU, Tokyo 158, JAPAN 